சு. சுஜா அ, *

அ தமிழ் இலக்கியத்துறை, சென்னைப் பல்கலைக்கழகம், சென்னை-600005, தமிழ்நாடு, இந்தியா.

\title{
Nandhi Kalambagam's Agam Songs and its Rhetoric
}

\author{
S. Suja a, * (D) \\ a Department of Tamil Literature, University of Madras, Chennai-600005, Tamil Nadu, India.
}

* Corresponding Author: kurinjipirai@gmail.com

Received : 20-11-2020 Accepted : 03-12-2020

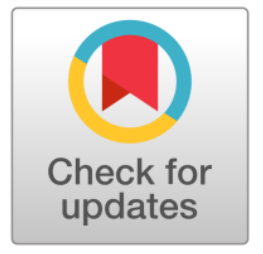

\begin{abstract}
:
As a result of the proliferation of Short literary composition genres (Prabandas), various catalog texts (Paattiyal) arose as a continuation of the tradition of finding literature and giving grammar explanations therefor. Panniru Paatiyal, Venpaa Paatiyal, Chidambara Paatiyal, Navaneetha Paatiyal, Prabandha Deepika, Ilakkana vilakkam, Thonnuul vilakkam etc. and even some grammar books that deal with five grammar forms (Ainthilakkanam) are involved in this grammatical endeavor and have given grammar to different numbers of Short Literary Compositions. These numerical differences record the development of the literature as a result of the passage of time. This number extends from 54 to 360. This genre of 96 Short Literary Works can be attributed to the fact that the number system operating in the set tradition is also applied to Short Literary Works and to be a permanent one. The name of the literary genre, Kalambakam, is given in various ways by dividing its name. There are various reasons for the mix of 18 types of elements (15-21), the proliferation of many types of compositions, and the mixing of Agappaadalkal (Agam songs). This can be explained by the fact that the name is derived from a variety of hybrids rather than one character. Nandikkalambakam, the first and foremost of the Kalambaka literatures, was sung with the third Nandi Varman of the Pallava dynasty as the Leader of the song. 25 years Nandi ruled from (847-872) with Kanchi as his capital, the Pallava dynasty and the wars fought to expand the territory of many Nandikalambaka songs. Although there are some differences in the view of Nandivarman's reign, it is accepted by scholars that he belonged to the ninth century and that Nandi Kalambakam, who led him to the song, and the ninth century. Even though this literature is in our school and college curriculum, its literary style beauty and glossary competency are unknown to the so called scholars too. So this article tries to explain the above said features of the Nandhi Kalambakam.
\end{abstract}

Keywords: Nandhi Kalambakam, Prabandas, Short literary composition, Agam Songs, Glossary Competency/ Rhetoric.

\footnotetext{
ஆசிரியர் குறிப்பு

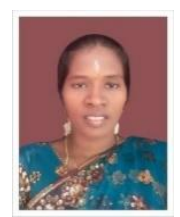

முனைவர் சு சுஜா இவர் சென்னைப் பல்கலைக்கழகத் தமிழ் இலக்கியத்துறையில் முதுமுனைவர் பட்டம் மேற்கொண்டுவருகிறார். ஆழ்ந்த சங்க இலக்கிய ஈடுபாடும் புலமையும் கொண்டவர். கனடாவில் நடைபெற்ற தமிழியல் மாநாட்டில் (Tamil Studies conference 2014) கலந்துகொண்டு கட்டுரை வழங்கியுள்ளார். மேலும் நாற்பதுக்கும் மேற்பட்ட ஆய்வுக் கட்டுரைகளையும் எழுதியுள்ளார். 'தமிழ்த் தொகுப்பு மரபு -
} 
எட்டுத்தொகைப் பனுவல்கள்', 'சங்கப் பனுவல்கள்: தொகுப்பு மரபு - திணைமரபு', 'தமிழியல்: மொழியும் வெளியும்' ஆகிய நூல்களை வெளியிட்டுள்ளார்.

\section{முன்னுரை}

நாயக்கர் ஆட்சிக் காலத்தில் நிலையான ஆட்சி ஏற்பட்டதன் விளைவாக இலக்கியப் படைப்புகள் சில தமிழுக்குக் கிடைத்தன. அவை முந்தைய இலக்கியங்களைக் காட்டிலும் அளவில் சிறிதாக இருந்தமையால் சிற்றிலக்கியங்கள் என்று அடையாளப்படுத்தப்பட்டன என்ற கருத்து பொதுவாகக் கூறப்படுவதுண்டு. சிற்றிலக்கியங்களுக்கான விதைகள் தமிழின் தொன்மை இலக்கணமாகிய தொல்காப்பியத்திலேயே இருந்தது என்பதால் அதற்கான ஆதாரங்களை வரிசைப்படுத்தமுடியும். தமிழில் சிற்றிலக்கியங்கள் என்று வழங்கப்படும் பெயர் மரபு வடமொழியில் பிரபந்தம் என்று சுட்டப்படுவது, சிற்றிலக்கியங்கள் பலவற்றையோ அல்லது பொருள் தொடர்புடைய / பொருள் தொடர்பற்ற பாடல்களையோ இணைத்துப் பார்க்கும் முறைமை காரணமாக, இணைக்கப்பட்டது அல்லது கட்டப்பட்டது என்ற பொருண்மையில் வழங்கப்பட்டது. பக்தி இலக்கியக் காலங்களில் பாடப்பட்ட வைணவம் தொடர்பான பாடல்கள் நாலாயிர திவ்ய பிரபந்தம் என்று வழங்கப்படுவதை இங்கு ஒப்புமை கருதி இணைத்துப் பார்க்க முடியும்.

பிரபந்த/ சிற்றிலக்கிய வகைமைகளின் பெருக்கத்தின் விளைவாக இலக்கியம் கண்டு அதற்கு இலக்கணம் இயம்பும் மரபின் தொடர்ச்சியாகப் பல்வேறு பாட்டியல் நூல்கள் எழுந்தன. பன்னிருபாட்டியல், வெண்பாப் பாட்டியல், சிதம்பரப் பாட்டியல், நவநீதப் பாட்டியல் பிரபந்த தீபிகை, இலக்கண விளக்கம், தொன்னூல் விளக்கம் முதலான பாட்டியல் நூல்களும் சில ஐந்திலக்கண நூல்களும்கூட இந்த இலக்கணம் இயம்பும் முயற்சியில் ஈடுபட்டு வேறுபட்ட எண்ணிக்கையுடைய சிற்றிலக்கியங்களுக்கு இலக்கணம் கூறியுள்ளன. இந்த எண்ணிக்கை வேற்றுமைகள் கால வளர்ச்சியின் காரணமாக ஏர்பட்ட இலக்கியத்தின் வளர்நிலையைப் பதிவு செய்கின்றன. 54 தொடங்கி 360 வரை (புலவர் இளங்குமரன், இலக்கியவகை அகராதி) இந்த எண்ணிக்கை நீட்சி அமைகிறது. சிற்றிலக்கிய வகைமை 96 என்பது நிலைத்த ஒன்றாக இருப்பதற்குத் தொகுப்பு மரபில் செயல்படுகின்ற எண்முறைமையைச் சிற்றிலக்கியங்களுக்கும் பொருத்திப் பார்த்தமையே காரணமாக அமைந்திருக்கமுடியும் (Ilankumaran Ira, Pulavar., 2012).

கலம்பகம் என்ற இலக்கிய வகைமையின் பெயர் குறித்து அதன் பெயரைப் பல்வேறு முறையில் பிரித்து அதற்கான பெயர் விளக்கங்கள் கூறப்படுவதுண்டு. 18 வகை உறுப்புகள் (15-21) கொண்டிருப்பது, பல யாப்பு வகைமைகள் விரவிவருவது, அகப்புறப் பாடல்கள் கலந்து பாடப்படுவது என்று கலப்பிற்குப் பல்வேறு காரணங்கள் கூறப்படுகின்றன. ஒரு தன்மைத்து அல்லாமல் பல்வேறு கலப்பினைப் பெற்றிருப்பதால் பெற்ற பெயராக இதனை விளக்க முடியும்.

கலம்பக இலக்கியங்களுள் முதலும் சிறப்பும் பெற்ற நந்திக்கலம்பகம் பல்லவ அரச மரபைச் சார்ந்த மூன்றாம் நந்திவர்மனைப் பாட்டுடைத் தலைவனாகக் கொண்டு பாடப்பட்டது. இருபத்தைந்து ஆண்டுகாலம் (கி.பி.847-872) வரை ஆட்சிபுரிந்த நந்தி காஞ்சியைத் தலைநகராகக் கொண்டு ஆட்சி செய்ததையும் பல்லவர் குலத்தைச் சேர்ந்தமையையும் ஆட்சி எல்லையை விரிவுபடுத்துவதற்காகச் செய்த போர்களையும் பற்றிய பதிவுகள் பல நந்திக் கலம்பகப் பாடல்களுள் இடம்பெற்றுள்ளன. நந்திவர்மனின் ஆட்சிக் காலம் பற்றிய கருத்தில் சில வேற்றுமைகள் இருந்தாலும் இவன் ஒன்பதாம் நூற்றாண்டிற்கு உட்பட்டவன் என்பதும் அவனைப் பாட்டுடைத் தலைவனாகக் கொண்ட நந்திக் கலம்பகமும் ஒன்பதாம் நூற்றாண்டினது என்ற கருத்தும் ஆய்வாளர்களால் ஏற்றுக்கொள்ளப்படுகின்றது. இந்த அறிமுகத்தோடு நந்திக் கலம்பகத்தில் இடம்பெற்றுள்ள தோழி, செவிலி, நற்றாய் கூற்றுப் 
பாடல்கள் குறித்தும் அதன் மொழிப்பயன்பாட்டுச் செழுமை குறித்தும் இக்கட்டுரையில் காணலாம்.

\section{அகப்பொருள் மரபும் நந்திக் கலம்பகப் பாடல்களும்}

பொதுவாக அகப்பொருள் மரபின் பின்னணியில் நந்திக் கலம்பகப் பாடல்களை ஆய்வுக்கு உட்படுத்தினால் அவற்றில் பல அகப்பாடல்கள் என்ற பாகுபாட்டிற்குள் வைத்தெண்ண முடியாதவையாகவே இருக்கின்றன. 'சுட்டி ஒருவர் பெயர்கொளப் பெறா மரபு', தலைவி நிலையில் பின்பற்றப்பட்டிருந்தாலும் பாட்டுடைத் தலைவனைக் கிளவித் தலைவனாக புறப்பாடல்களைப் போல அகப்பாடல்களிலும் ஆசிரியர் சிறப்பிப்பது இங்கு இதற்கான காரணமாக உள்ளது. 'வேம்பு தலையாத்த நோன்காழ் எஃகம்' என பாட்டுடைத் தலைவனின் அடையாள மாலை இலக்கியத்திற்குள் இடம்பெற்றதன் அடிப்படையில் அதனைப் புறமாக வைத்தெண்ணும் நிலையில் மூன்றாம் நந்திவர்மப் பல்லவனின் பெயரும் தொண்டை மாலையும் தலைவியால் விரும்பப்படுவதாகத் தொடர்ச்சியாகச் செவிலியாலும் தோழியாலும் கூறப்படுகின்றன. இவ்வாறல்லாமல் நந்தி, பாட்டுடைத்தலைவனாக மட்டும் இடம்பெறுவதாகவும் இக்கலம்பகத்தில் பாடல்கள் உள்ளன. இந்த வகையில் மட்டுமன்றி கைக்கிளைப் பின்னணியிலும் - தலைவனின் மாலையை வேண்டுதல், தலைவியை முயங்கும்படிக் கூறுதல் என்ற நிலையில் இப்பாடல்கள் அகத்தோடு சேர்த்தெண்ணத்தகாதவை. பிற்கால அகப்பொருள் இலக்கண வளர்ச்சியின்படி இவை அகப்புறமாக பாகுபடுத்தத் தக்கவை.

தோழி, செவிலி, நற்றாய் கூற்றாக நந்திக் கலம்பகத்துள் அமையப்பெற்றுள்ள முப்பதுக்கும் மேற்பட்ட பாடல்கள் கூற்றுநிலையில் களவுகாலத்தைச் சேர்ந்தவையாக உள்ளன. களவு, கற்பு என்ற இருவகைப் பகுப்புகளுக்குள் அறத்தொடு நிற்றல் வரையிலான கிளவித் தொகைகள் களவுக்குள்ளும் அதனைத்தொடர்ந்து நிகழும் கூற்றுகளைக் கற்பினுள்ளும் தொல்காப்பியம் உள்ளிட்ட பழைய இலக்கண நூல்கள் குறிப்பிடுகின்றன. ஆனால் நம்பியகப்பொருள் உள்ளிட்ட பிற்கால இலக்கண நூல்கள் களவியலுக்குப் பின்னர் வரைவியலையும் அதன் பிந்தைய கூற்றுகளைக் கற்பியலுக்குள்ளும் வைக்கின்றன. திருமணத்திற்குப் பிந்தைய இல்லற வாழ்வு பற்றிய பதிவுகள் நந்திக் கலம்பகத்தின் தோழி, செவிலி, நற்றாய் கூற்றுப் பாடல்களில் இல்லாத நிலையில் இவற்றை முற்றும் முழுதாகக் களவுப் பாடல்களாகவே அடையாளப் படுத்தலாம். பாட்டுடைத் தலைவனே கிளவித்தலைவனாகவும் இடம்பெறுவதாலும், அவனின் வீரம், வெற்றி, கொடை, தோற்றப்பொலிவை முதன்மைப்படுத்தி எடுத்துக் காட்டுவதையே நோக்கமாகக் கொண்டதாலும் கற்புப் பாகுபாட்டினுள் அடங்குபவையாகப் பாடல்களைப் பாடமுடியாது என்பதை நாம் இங்கு விளங்கிக்கொள்ள முடியும்.

\section{நந்திக்கலம்பகச் செய்யுள்களின் பொருட்பன்முகத்தன்மை}

கூற்றுநிலைப் பாடல்கள் பொருள் கொள்ளும் முறையில் பன்முகத்தன்மையைப் பெற்றுவிடுவதற்குச் சங்கத் தொகைப் பாடல்களிலிருந்தே நாம் சான்றுகாட்ட முடியும். 'தலைவி கூற்று, தோழி கூற்றுமாம்', 'தோழி கூற்று, செவிலி கூற்றுமாம்' என்பன போன்ற கூற்றுக் குறிப்புகளை எட்டுத்தொகைச் செய்யுள்களுக்கு பேராசிரியர், இளம்பூரணர், நச்சினார்க்கினியர் உள்ளிட்ட முந்தைய உரையாசிரியர்களும் உ.வே.சா உள்ளிட்ட பிற்காலப் பதிப்பாசிரியர்களும் வழங்கியிருப்பதை இங்கு கவனம்கொள்ள வேண்டும். அப்படியான பன்முகத்தன்மையை நந்திக் கலம்பகத்தின் இருவேறு பதிப்புகளை ஒப்பு நோக்கிப் பார்க்கும் போதும் காணமுடிகிறது. சான்றாக, 
- 'விரவாத மன்னரெல்லாம்' எனத் தொடங்கும் பாடல் மர்ரே பதிப்பில் மாலை பெறாது வருந்துதல் என்று குறிப்பிடப்பட்டுள்ளது. ஆனால் கழகப்பதிப்பில் தோழி தலைவனை இயற்பழித்ததாக உள்ளது.

- இது போலவே 'பகரங்கொள் நெடுந்திவலை' பூவை நிலையாக மர்ரே பதிப்பிலும் தோழி கூற்றாகக் கழகப் பதிப்பிலும் இடம்பெற்றுள்ளது.

- நங்கள் கோத் தொண்டை வேந்தன்' பாடல் நந்தியின் உலாக் கண்ட மகளிர் கூற்று மர்ரே. இதுவும் அது(தோழி கூற்று) - கழகம்.

- 'கோவேம் மாலை' நந்தியின் புகழ் - மர்ரே. தோழி தலைவியின் நிலை கிளத்தல் கழகம்.

- 'காவி அனந்தம்' தலைப்பில்லாமல் தனிப்பாடல் - மர்ரே. தோழி தலைவியின் நிலைகண்டு வருந்தியுரைத்தல் - கழகம்.

- 'மொழிஆர் தொண்டை' தோழி கூற்று - மர்ரே. தலைவன் கார்கண்டு பாகனொடு கிளத்தல் - கழகம்.

- ‘ஒரு கோமகன்' நந்தி என்ற பாடல் மர்ரே பதிப்பில் தோழி கூற்றாகவும், கழகப் பதிப்பில் தலைவி இரங்கல்: நிலவை வெறுத்துரைத்தல் என்பதாகவும் உள்ளது.

பதிப்பு ஒப்பீடு அல்லாமல் தனியொரு பாடலை பொருள்கொள்ளும்போதும் இந்தத் தன்மையை நம்மால் நந்திக் கலம்பகத்தில் காணமுடியும்.

மொழியார் தொண்டைப் பன்மலர் முற்றும் தெருவந்து

விழியாள் என்றும் மேனி வெளுத்துற மெலிவாளே

ஒழியா வண்கைத் தண்ணருள் நந்தி தன் ஊர்மட்டோ

வழியாம் தமரக் கடல்வட் டத்தொரு வண்கோவே

(ந.க.43, தோழிகூற்று - மர்ரே. 47, தலைவன் கார் கண்டு பாகனொடு கிளத்தல் -கழகம்)

நிற்காத / மாறாத கொடைத் தன்மையையும் இரக்கப் பண்பும் கொண்ட நந்தி மன்னனின் மாமல்லபுர நகரில் மட்டும்தானா அல்லது கரிய கடல்சூழ்ந்த தான் செல்லும் வழிகளிலெல்லாம் மேகங்கள் சூழ்ந்துள்ளனவா? என் விழிபோன்றவள் புகழத் தகுந்த தொண்டை மலர்களும் பலவகை மலர்களும் நிறைந்திருக்கின்ற மாமல்லபுரத் தெருவிடத்து வந்து நின்று இவற்றைப் பார்த்து மேனி வெளுத்து என்றும் மெலிவடைவாளே என்ற பொருளில் பாடல் உள்ளது.

இதனைத் தோழி கூற்றாகப் பொருள் கொள்ளும்போது, விழி போன்றவளாகத் தலைவி தோழிக்கு இருக்கிறாள். தலைவன் கூறுவதாகப் பொருள் கொள்ளும்போது விழிக்குள் இருப்பவளாகத் தலைவியும் மேகம் செல்லும் வழி தலைவன் செல்லும் வழியாகவும் கொள்ளப்படுகிறது.

\section{தலைவி கூற்றா? தோழி கூற்றா?}

விரவாத மன்னரெலாம் விண்ணேற வெள்ளாற்று வெகுண்டோன் தொண்டைக்கு

இரவாத பரிசெல்லாம் இரந்தேற்றும் பாவைமீர் எல்லீர் வாடை

வரவாதை உற்றிருந்து வருந்துவார் பலர் என்றும் வாழி வாழி

பரவாதை நந்திசெங்கோல் இதுவாகில் அதுபார்க்கும் பரிசு நன்றே 
(ந.க.19/23, மர்ரே: மாலை பெறாது வருந்துதல், கழகம்: தோழி தலைவனை

இயற்பழித்தல்)

மாலைபெறாது வருந்தும் தலைவியின் நிலையாகவும் தோழி தலைவனின் இயற்பழித்துரைப்பதாகவும் இப்பாடல் பொருள்கொள்ளப்படுவதற்கான தன்மைகைத் தன்னிடத்தே கொண்டுள்ளன.

தன்னோடு சேராத பகையரசர்களை வீரசுவர்க்கம் அடையும்படி வெள்ளாற்றுப் போரில் சினம்கொண்டு அழித்தவனின் தொண்டை மாலையை இரவாத பரிசு எலாம் - கேட்கக் கூடாத/ கேட்கும் தன்மைக்கு ஒவ்வாத தன்மைகளில் எல்லாம் இரப்புரை கூறி வேண்டுகின்ற தோழிகளே, பிறருக்குத் துன்பத்தைக் கொடுக்கின்ற நந்தி மன்னனின் செங்கோல் சிறப்பு இப்படிப்பட்டதாக இருக்கும் என்றால் அப்படிப்பட்ட செங்கோன்மையை எதிர்பார்த்து இருக்கும் நிலை நல்லது. இரவுப்பொழுதுகளில் (ஈர்) வாடைக்காற்று (வருத்துகின்ற வாடை, ஈரமான வாடை) வர அதனால் துன்பமடைந்து வருந்துபவர்கள் பலரும் எக்காலமும் வாழ்வார்களாக.

நந்தியின் தொண்டை மாலை வேண்டிய பெண்களுக்கு இரவு வாடை வருத்தம் தருவதாக இருப்பதால் அதனை மாற்றும்படியாக மாலை வழங்கி வருத்தும் வாடை வராது தடுத்தலே செங்கோல் சிறப்பு. அவ்வாறல்லாமல் பகைவரைப் போலவே தன் மீது அன்பு கொண்டவர்களுக்கும் துன்பம் தருவது எப்படிச் செங்கோன்மையாகும் என்று தலைவனின் இயல்பை சொல்லாது சொல்லி தோழி இயற்பழித்ததாகவும் தலைவி மாலை பெறாமல் வருந்துவதாகவும் இப்பாடலைப் பொருள்கொள்ள முடியும்.

\section{பதிப்பு வேறுபாடுகள்}

மேற்குறிப்பிட்டது போலவே நந்திக்கலம்பகத்தின் பாடல் எண்களும் மாறி மாறியே உள்ளன. காவி அனந்தம் எடுத்தான், மதன் கைக் கரும்பெடுத்தான் என்ற பாடல் கழகப் பதிப்பில் 'இப்பாட்டு சில சுவடிகளில் காணப்படுகின்றது' என்ற குறிப்போடு 82ஆம் பாடலாக இடம்பெற்றுள்ளது. பல பிரதிகளை ஒப்புநோக்கிப் பதிப்பிக்கப்பட்ட மர்ரே பதிப்பில் இப்பாடல் தனிப்பாடல்களுக்குள் இடம்பெற்றுள்ளது. தனிப்பாடல் எண்ணிக்கையில் இந்த வேறுபாடு உள்ளது. முன்னுள்ள பாடலின் கூற்றோ, உறுப்போ பின்னைய பாடலுக்கும் உரியதாக இருக்கும்போது ‘துவும் அது’ என்ற குறிப்பு இடம்பெறுவது சுவடிக்குறிப்பின் தொடர்ச்சியாகப் பதிப்புகளிலும் பின்பற்றப்படுகிறது. இந்தத் தன்மை பாடலின் தவறான/ மாறுபட்ட பொருள்கோடலுக்கு வகைசெய்யும் என்பதை நாம் இங்கு மறந்துவிடக்கூடாது. அந்தாதி முறையில் பாடப்பட்ட ஒரு இலக்கியத்திற்குள் இத்தகைய குழப்பம் நேரக் காரணமாக இருப்பது ஒரு குறைப்பாடல். 'ஆகாது போக மயில் வினைத்தகன் றலவன்கை' என்ற பாடலின் இறுதி இரண்டு வரிகள் கிடைக்கப்பெறாததால் எந்தப் பாடலையும் இடையில் இணைக்க முடியும். அப்படி இணைத்தால் அடுத்தப் பாடலின் தொடக்கமாக முன்அமையும் பாடலின் இறுதி அமையவேண்டும். எனினும் நந்திக் கலம்பகத்தின் பல பாடல்களில் சொல்லோ, சீரோ அந்தாதியாக அமையாமல் அசைகளும் அந்தாதியாகக் கொள்ளப்பட்டுள்ளமையால் இத்தகைய மாற்றங்கள் நிகழ்ந்துள்ளன. நந்திக் கலம்பகத்தை வாசிப்பிற்கு உட்படுத்தியவர்கள் பிரதிக்குள் நிகழ்த்திய ஊடாட்டமாக இதனை நாம் பார்க்கவேண்டும்.

\section{நந்திக்கலம்பகத்தில் மொழியாளுமை}

நந்திக்கலம்பகத்தினை சிறந்த இலக்கியமாகக் கருதுவோரும் செவ்வியல் இலக்கியங்களுக்கு இ இணையான இடத்திற்குள் அதனை வைத்தெண்ணுவதில்லை; அது சரியும் அல்ல. என்றாலும் நந்திக் கலம்பகத்திற்குள்ளும் மொழிநிலையில் நாம் கவனம்கொள்ளவேண்டிய சில இடங்கள் உள்ளன. நந்திக்கலம்பகத்தின் சிறந்த சொற்களைக் கண்டறியும் முயற்சி என்பதையும் தாண்டி அதன் மொழியியல் எல்லைக்குள் நின்று 
மொழிப்பயன்பாட்டின் செழுமையை கோடிட்டுக் காட்டும் முயற்சியாக இங்கு சில சொற்கள் எடுத்துக் காட்டப்படுகின்றன.

- தொழில் செய்பவன் தொழிலாளி, உளவு பார்ப்பவன் உளவாளி, என்பது போல ஆள்பவனை ஆளி என்று இவ்விலக்கியம் பதிவுசெய்துள்ளது (ந.க.51/55).

- பெண்கள் காதுகளில் அணியும் அணிகலனை சங்க இலக்கியங்கள், ஓலை, குழை முதலான சொற்களில் குறிப்பிட்டுள்ளன. அ அந்த அணிகலன் தெடக்கத்தில் இலைகளாலும் மலர்களாலும் பொன்னாலும் அணியப்பட்டன. இக்கலம்பகத்திலும் பொன்னால் செய்யப்பட்ட காதணி பற்றிய குறிப்பு இடம் பெற்றுள்ளது (ந.க.மர்ரே.த.பா.14). ஆனால் அதனை இன்று நம் புழக்கத்தில் அதிகமாகப் பயன்படுத்தும் சொல்லான தோடு என்ற சொல்லால் நந்நிக் கலம்பகத்தின் பாடல் குறிப்பிட்டுள்ளது (Punnaivananadha Mydhaliyar. P.C., 1995).

- இதுபோலவே விபரீதம் என்ற சொல்லின் இசை காரணமாகக் குறுகிய வடிவமான விபரிதம் என்ற சொல் இடம்பெற்றுள்ளது (ந.க.39/43). மணிமேகலையிலும் பயன்படுத்தப்பட்டுள்ள இச்சொல் மாறுபட்டிருக்கும் தன்மை என்ற பொருளில் நந்திக் கலம்பகத்தில் இடம்பெற்றுள்ளது

- வாளைக் குறிக்கச் சுரிகை என்ற சொல் நான்காம் பாடலில் பயன்படுத்தப்பட்டுள்ளது. பழைய இலக்கியங்களுள் பெரும்பாணாற்றுப்படையில் மட்டுமே இதே (73)

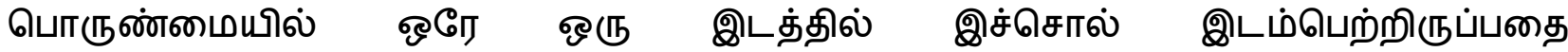
அறியமுடிகின்றது.

- ஓலை என்ற சொல்லால் அரசனின் கடிதத்தை/ திருமுகத்தைக் குறிப்பிடும் வழக்கு இருந்தமை அறியப்பட்ட செய்தியே. நந்திக்கலம்பகத்திலும் ஒருபாடலில் (ந.க.11) ஓலை குறிப்பிடப்பட்டுள்ளது. அந்த ஓலை விடைமண்பொறி ஓலை. இந்த ஓலை நந்தி மன்னனின் கொடியில் உள்ள விடை என்னும் எருதின் உருவம் மண்மூலம் பொறிக்கப்பட்ட ஓலையாக உள்ளது. பொறி என்கிற சொல் பல்வேறு பொருண்மைகளில் பயன்படுத்தப்பட்டிருந்தாலும் ஒரு அகநானூற்றுப் பாடலிலும் (77:8), ஒரு புறநானூற்றுப் பாடலிலும் (58:3) இதே பொருண்மையில் இடம்பெற்றுள்ளது. ஆனால் அங்கு இச்சொற்றொடர் மூலம் விளங்கிக் கொள்ளப்படும் பொருளில் இல்லை என்பது குறிப்பிடத்தக்கது.

- வட்டிப்பன் என்ற செல் ஆணையிடுதல் என்ற பொருளில் (ந.க.63) பயன்படுத்தப்பட்டுள்ளது. கலித்தொகையின் 136ஆம் பாடலில் இச்சொல் தாயவிளையாட்டரங்கைக் குறிப்பிடுகின்றது.

- வாதை என்ற சொல்லும் நந்திக்கலம்பகத்தில் இடம்பெற்றுள்ளது (ந.க.81). தமிழ் ஆக்ஸ்போர்டு அகராதியின் வலைத்தளத்தில் அருகிவரும் வழக்குச் சொல்லாக இச்சொல் குறிப்பிடப்பட்டுள்ளது. துன்பம் என்ற பொருள் தருகின்ற இச்சொல் தேவாரத்தில் ஒரு இடத்தில் இடம்பெற்றுள்ளது (தேவா.570:2). தென்மாவட்டங்களில் துன்பத்தைக் கொடுக்கின்ற தீயசக்திகளை வாதை என்று குறிப்பிடும் வழக்கம் இன்றும் உள்ளது இங்கு குறிப்பிடத்தக்கது.

- மடல் குறித்த கருத்தியல்கள் காலத்திற்குக் காலம் மாற்றம்பெற்றதைப் போல நந்திக் கலம்பகமும் ஒருவகை மாற்றத்தைப் பதிவுசெய்துள்ளது. நந்தி மன்னனை எதிர்த்த பகைவர்கள் மடல் ஏறி உயிர்விடுவதாக ஒரு புதுமைக் கற்பனையை (ந.க.55/59) என்ற பாடலில் ஆசிரியர் பதிவுசெய்துள்ளார்.

- காலமறிந்து வினை செய்யவேண்டிய கடமையுடையவர்களான உழவர்களை காலவினை வாணர் என்று பதிவுசெய்வது (ந.க.57) புலனுழுதுண்மார் என்ற சங்கத் 
தொடரை நினைபடுத்துகிறது. ஒரு செயலைச் செய்பவரை அச்செயல் செய்யப்படும் திறன் உணர்ந்து குறிக்கும் இந்த வழக்கு கவனிக்கத் தக்கது.

- கண் சிம்புளியாநோய் என்ற ஒரு புதிய நோயைக் குறிப்பிடுகிறார் நந்திக்கலம்பக ஆசிரியர். நோய் புதிதல்ல; சொல்லப்படும் சொல்தான் அரிய வழக்கு (ந.க.72), நாம் மறந்துவிட்ட வழக்கு. இச்சொல் சுந்தரர் தேவாரத்தில் இடம்பெற்றுள்ளது. 'சிம்மாந்து சிம்புளித்துச் சிந்தையினில் வைத்துகந்து' கண் மூடியும் மூடாத நிலையாகப் புலவர் செல்வகணபதி இதற்குப் பொருள் கூறுகிறார் (Gnyanasambandham,1976)

- பழுத்தல் என்ற பண்பை - தொழிலை செய்யவைப்பதை அல்லது அதன் பிறவினை வடிவத்தைக் குறிப்பதற்கு ஒரு புதுமையான சொல்லைப் பயன்படுத்தியுள்ளார் ஆசிரியர். இந்த வடிவத்தைக் குறிப்பதற்குத் தற்போது பழுக்க வைத்தல் என்ற சொல் பயன்படுத்தப்படுகின்றது (ந.க.68). இ இங்கு வைத்தல் என்ற துணைவினும் இணைக்கப்பட்டாலே இப்பொருள் தருகின்றது. ஆனால் பழுப்பித்தல் எளிமையாகப் பொருளை விளக்கும்படியாக இக்கலம்பகத்தில் இடம்பெற்றுள்ளது (Rajam S Marre., 1960).

- குட்டு என்ற சொல் ஊருணியைக் குறிக்கப் பயன்படுத்தப்பட்டுள்ளது (ந.க64/68).

\section{நந்திக் கலம்பகத்தின் சொற்சுவையும் பொருட்சுவையும்}

நந்திக் கலம்பகத்திற்குள் படித்துச் சுவைக்கத் தகுந்த பல பாடல்களும் இடம்பெற்றுள்ளன. எதுகை மோனையோடுகூடிய சொல்விளையாட்டுப் பாடல்களும் சந்தச் சிறப்பு மிகுந்த பாடல்களும் இடம்பெற்றுள்ளன. அத்தகைய பாடல்களுக்குக் காட்டாக,

கோட்டை இடித்தகழ் குன்றாக்கிக்

குன்றகழ் ஆக்கித் தெவ்வர்

நாட்டை மிதிக்கும் கடாக்களிற்

றான்நந்தி நாட்டினில் பொன்

தோட்டை மிதித்தந்தத் தோட்டூடு

பாய்ந்து சுருள் அளகக்

காட்டை மிதிக்கும் கயற்கண்ணி

யோசுரம் கால்வைப்பதே (ந.க.107, செவிலி இரங்குதல்)

பகைவர்நாட்டின் மதில்களைத் தனது கொம்புகளால் இடித்துக் குன்றுபோலாக்கி, மலைபோல் உயர்ந்த மதில்களை அகழியாக ஆக்கி, பகைவர்களின் நாட்டினுள் செல்கின்ற ஆண்யானையை உடையவனான நந்தி மன்னனின் நாட்டில் பொன்னால் ஆன காதணியைக் கடந்து சென்று தோடுகளின் ஊடாகப் பாய்ந்து சென்று சுருண்ட கூந்தல் காடுவரை போகின்ற கெண்டைமீன் போன்று கண்களையுடைய என் மகளோ, பாலைநில வழியில் கால்வைத்து நடந்து செல்வது.

துயக்குவித் தான்துயில் வாங்குவித் தான்துயில் வித்திவளை

வயக்குவித் தானுள்ளம் வஞ்சனை யான் மலர்க் காவகத்து

முயக்குவித் தான்துகில் வாங்குவித் தான்முனம் நின்றிவளை

வயக்குவித் தானந்தி மானோ தயனென்று வட்டிப்பனே

(ந.க.67, பாங்கி செவிலிக்கு அறத்தொடுநிற்றல்)

நந்தி என்னும் பெயருடைய மானமிக்கவன் நமது ோழியை சோர்வுபடுத்தினான், அவளது உறக்கத்தையும் பறித்தான் மீண்டும் தானே துயில்வித்து வஞ்சனையால் தன்வசப்படுத்தினான். மலர்ச்சோலையிடத்து தன்னைத் தழுவும்படிச் செய்தான், ஆடை நெகிழச் செய்தான், இவள் முன்னே நின்று இவளுக்கு மயக்கம் உண்டாகும்படிச் செய்தான் என்று நான் ஆணையிட்டு உறுதியாகக் கூறுவேன் ஆகிய இரண்டு பாடல்களைக் கூறலாம். 
நந்திக்கலம்பகத்தின் பாடல்களுள் பல பயில்வுப் பாரம்பரியத்தின் வெளிச்சம் படாமல் பயிலப்பட்ட பாடல்களே மீண்டும் மீண்டும் பாடத்திட்டத்திற்குள் சேர்க்க்பட்டிருப்பதையும் மேற்குறிப்பிட்ட பாடல்கள் நமது பரந்த இலக்கியவெளிக்குள் நந்திக்கலம்பகமும் பிற கலம்பக இலக்கியங்களும் எத்தகைய ஆழத்தை எதிர்நோக்கியுள்ளன என்பதை நமக்கு எடுத்துக் காட்டுபவையாக உள்ளன.

\section{முடிவுரை}

கலம்பக இலக்கிய வமையில் படைக்கப்பட்ட பல்வேறு நூல்களுள் முதலும் முடிவுமாக எளிமையும் இனிமையும் கொண்ட நூலாக நந்திக்கலம்பகம் உள்ளது. பல்வேறு உறுப்புகளைக் கொண்டு பாடப்படும் இலக்கிய வகைமையாக இருந்தாலும் தொடர்ச்சியாக, அளவுகோலில் கோடிடுவது போன்ற ஒரு அமைப்பு முறையைக் கொண்டிராமல் கலவையாக உறுப்புகளும் பொருண்மையும் விரவி வரும்படியாக இவ்விலக்கியம் பாடப்படுள்ளது. இதனால் பிரதியைக் கலைத்துப்போட்டு வாசிக்கின்ற பின்நவீனத்துவ வாசிப்புத் தன்மையைக் கொண்ட சிறந்த பிரதியாக நந்திக் கலம்பகத்தை நாம் அடையாளப்படுத்தமுடியும்.

\section{References}

Ilankumaran Ira, Pulavar., (2012) Ilakkiyavagai Agarathi, Manivasagar Padhippagam, Chennai, Tamil Nadu, India.

Punnaivananadha Mydhaliyar. P.C., (1955) Nandhi Kalambakam, with Se. Re. Ramasamippillai Commentary, Tirunelveli Thennindhiya Saiva Siddhandha Noorpadhippu Kazhagam, Chennai, Tamil Nadu, India.

Rajam S Marre., (1960), Nandhi Kalambakam, Chennai, Tamil Nadu, India.

Gnyanasambandham (1976), Dharumayadheena thingal Idazh, Malar 36, Idhazh11.

Funding: NIL

Acknowledgement: NIL

Conflict of Interest: NIL

\section{About the License:}

\section{(c) (1)}

Attribution 4.0 International (CC BY 4.0)
(C) The author 2021. The text of this article is licensed under a Creative Commons Attribution 4.0 International License 